\title{
How Big Is an Acupoint?
}

\author{
Lei $\mathrm{Li}^{1 *}$, To Yau', Chuen-Heung Yau' \\ ${ }^{1}$ School of Chinese Medicine, The University of Hong Kong, Hong Kong, China \\ ${ }^{2}$ School of Chinese Medicine, Hong Kong Baptist University, Hong Kong, China \\ Email: llie@hku.hk
}

Received July 19, 2012; revised September 21, 2012; accepted October 2, 2012

\begin{abstract}
The structure and size range of an acupoint were investigated thoroughly according to the expositions of ancient classics. It was revealed that the ambiguous way of thinking by the ancient Chinese had a great impact on the formation and development of the acupoint-concept. Hence all descriptions of an acupoint's structure and size range have the characteristics of ambiguity. The structure and size range of an acupoint are determined not only by the outlook of depression and blood vessel areas, but also its relationship with other acupoints in its vicinity. The different manipulations of puncturing recorded in the Yellow Emperor's Canon of Medicine also show the ambiguous nature of the structure and size range of an acupoint. In theory, an acupoint has been characterized as various forms, which should not be limited to a mere round dot shape.
\end{abstract}

Keywords: Acupoint; Acupoint Structure; Acupoint Size Range

\section{Introduction}

How to define the size range has become a major concern when it comes to the study of an acupoint, for it is closely related to clinical applications of acupuncture therapy. However, prior to deciding the size of an acupoint, its structure first needs to be clarified. Since an acupoint is a spot for the flow of Qi and blood, reflecting symptoms of diseases, and assisting in syndrome differentiations in the treatments of pathogens, its existence has appeared to be objective, and undoubtedly has the material structure. In fact, the formation of acupoints is actually a creation of the ancient Chinese based on an ambiguous logical concept. Therefore, an acupoint's structure is not limited to shape, but is also closely related to the character of its allying function. Nowadays, with all the state-of-art science and technologies, it is still impossible to explain the actual structure of acupoints. However, that does not stop us from attempting to theoretically explain the structural ambit of acupoints through analyzing related discussions in ancient literatures and classics.

\section{The Acupoint Is Relatively Hollow Stereoscopic Structure}

"Acupoint" implies a hole. Most of the acupoints are located in the depressions between bones and joints or muscular interstices. Thus they structurally resemble three-dimensional hollows. As the nature of the depresssions between bones and joints or muscular interstices varies, the presumed sizes of acupoints are comparatively different.

In view of the origin of acupoints, their formations are closely related to the blood vessels. In early ancient times, acupuncture meant using a stone needle to pry out blood from the pain points of a bodily part, and an acupoint meant the location of a blood vessel where the stone needle was to be applied. The Yellow Emperor's Canon of Medicine includes a large number of related discussions. In The Plain Conversation: Discussion on Treatment of Lumbago with Acupuncture, it explained the methods for locating acupoints, for example, "Needling Jiemai (branch of Foot-Taiyin channel)", "Needling Tongyin channel (collateral of Foot-Shaoyin channel)", "Needling Yangwei channel", "Needling Feiyang channel", "Needling Sanmai", "Needling the channel inside of muscles" and etc [1]. At that time, puncturing blood vessels was equivalent to the puncturing of acupoints, and the sites of the blood vessels were exactly the sites for acupoints. In addition, from the naming of Daying (ST5), Renying (ST9), Chongyang (ST42), Taichong (LR3), Chimai (TE18) and so on, we found an obvious trace of transformation from "vessels" to "acupoints". The size of an acupoint is therefore also classified according to the appearance of blood vessels.

In the Yuan Dynasty, Dou Hangqing mentioned when defining the locations of acupoints, "At the Yang level lateral to the muscle and bone, depressions as existent; at the Ying layer between hollow and poles, corresponding to the artery [2]." In the Ming Dynasty, Xu Feng further 
explicated, "Yang level is where all Yang channels located, such as Hegu (LI4), Sanli (ST36) and Yanglingquan (GB34), with feeling depressions lateral to the middle bone as the real locations; Yin layer is where all Ying channels are located, such as Jimen (SP11), Wuli (LR10) and Taichong (LR3), in the middle of the muscles where arteries have to be felt, and those are the real points [3].”

Acupoints are closely related with the blood vessels, which no doubt reflected the hollow characteristics of acupoint structure.

\section{The Acupoint Possess Diverse Structural Features}

In modern acupuncture, acupoint is merely considered as a round dot-structure, where the distributions and measurements of 14 meridians, including Meridian points, Extra points and Ashi points over the whole body are considered as one type. In fact, this is untrue. The interstices between bones and joints, and the depressions between tendons and flesh actually come with different shapes. Acupoints located at the Large Collaterals, Small Collaterals, Floating Collaterals, arteries and other kinds of blood vessels varies in shape. Therefore, a diversified three-dimensional structure has to be seen as a distinctive character of acupoint.

For instance, Jingming (BL1) is defined in the $A-B$ Canon of Acupuncture and moxibution: chapter 3 section 10 as "Lateral to the inner canthus of the eye" [4]. Here, we see that the acupoint does not locate on top of a dot; but at a long and narrow hollow between the eyeball at the inner canthus of the eye and the orbit. Hence, it is a hollow structure in narrow rectangular shape. Some newly invented names, such as Superior Jingming, Medial Jingming, Lateral Jingming, Jingguang, Dongming1, Jianming4 are simply different names to describe the same Jingming (BL1). Any acupoint located in long and narrow hollows at the medial angle of the inner canthus of the eye, and between medial wall of the orbit and the eyeball shall fall into the same category as the acupoint structure of Jingming (BL1).

Weizhong (BL40) stated as "In the popliteal fossa, as the He-Sea, found with the knee bent" (Spiritual Pivot: Discussion on Acupoints) [5], and "In the popliteal fossa, near the arterial line." (A-B Canon of Acupuncture and moxibution: chapter 3 section 35 ). This acupoint is actually located in the popliteal fossa, correlated with the politeal vein and artery in its deeper layer, and medial from Weiyang (BL39). Thus, the structure and ambit of Weizhong (BL40) have been included in a large portion of popliteal fossa, and in fact, it is a rhombus with hollow structure, especially when it comes to puncturing blood from this acupoint.
Taichong (LR3) was situated "In the depression, 2 Cun above Xingjian (LR2)” (Spiritual Pivot: Discussion on Acupoints), "2 Cun posterior to the lateral side of the big toe, or 1.5 Cun in the depression” (A-B Canon of Acupuncture and moxibution: chapter 3 section 31 ), and "Half Cun posterior to Xingjian (LR2)" (Collective Compendium on Acupuncture and moxibution: chapter 3) [6]. In fact, Taichong (LR3) is located on the dorsum of the foot, between the first and second metatarsal bones, in the depression distal to the junction of two bones, underneath the Taichong pulse (the first dorsalis pedis artery of the first metatarsal bone). Its positioning is based on the pulse of the vessel, and thus the way of measurement varies: 2 Cun above the joint, 1.5 Cun above the joint, and half Cun posterior to Xingjian (LR2). From here we can see that the complete portion of long and narrow depression on the dorsum of the foot between the first and second metatarsal bones has been interpreted as Taichong (LR3), and is under its structural ambit.

Acupoint is not limited to a round dot-shaped spatial structure. Some acupoints can be dots while some can be of any other shapes. As the location of an acupoint varies, its shape and structure also differs. Starting from the late Ming Dynasty, there was a rise of Pediatric Tuina Therapy, which created some designated acupoints, some were dots, some were in lines and some were considered to be surfaces. Of course, the creation of line-shape acupoints and surface-shape acupoints originated from the different manipulation methods of Tuina when which applied to human bodies. However, it does demonstrate that the ancients had not limited their understanding of acupoints to mere round dots.

\section{The Size of an Acupoint Depend on Its Location}

As each acupoint has its own shape and structure, the size range will be different from one another. Some acupoints are situated at a specific narrow position, and their structural ambits are usually not huge. Typical examples are the twelve Jing-Well points, Suliao (GV25), Ciliao (BL32), Zhangmen (LR13) and Jingmen (GB25).

However, the size of an acupoint appearing on the surface of a body may not necessarily correspond to its inner structural ambit. For instance, Dicang (ST4) is located at the angulus oris, but it is usually puncturing obliquely towards Jiache (ST6), and thus its structural ambit is not limited to the angulus oris. Another example, Yaoqi (EX-B9) is positioned 2 Cun superior to the tip of the coccyx, in the depression of the sacral angle, but it is required to puncture horizontally upwards for 2 to 3 Cun, that means its structural ambit is not limited to the depression in the sacral angle.

The size of an acupoint has been closely related to 
other acupoints in its neighborhood, and this seems to be a common understanding. For example, Chengfu (BL36), Xinmen (BL37), Huantiao (GB30), Zusanli (ST36) and Chengjin (BL56) are further apart from their neighborhoods, so that their structural ambits are relatively larger. When the surrounding acupoints are larger in number, the captioned acupoint will surely be limited to a smaller ambit. For example, Shenting (GV24), Shangxing (GV23) and Meichong (BL3) are close to each other; and Yingjiao (CV7), Qihai (CV6), Shimen (CV5), Zhongzhu (KI15) and Siman (KI14) are closely located; and thus all their structural ambits are fairly limited.

Some structural ambits of acupoints have been changed from time to time. For instance, in the A-B Canon of Acupuncture and moxibution, Chimai (TE18) was positioned as "At the vein on the back of the ear which looks like a chicken talon”, while Luxi (TE19) was placed “At the vein on the back of the ear", where both acupoints had been described "On the back of the ear" and "At the vein" as their standard measurements, and their therapeutic intents are also similar. In fact, this is a practice to split one acupoint into two based on the position of the blood vessel. Another example is Baihui (GV20), which is vertically superior to the auricular apex, at the median point on top of the head. In the Peaceful Holy Benevolent Prescriptions, Baihui (GV20) was positioned "At the depression in the center of the head" [7]. Since the way of puncturing this acupoint was inserting the needle horizontally along the skin, this triggered the question of puncturing the needle towards its posterior, anterior, lateral or medial sides, and the solution came with the formation of Sishencong (EX-HN1). The Peaceful Holy Benevolent Prescriptions had stated, "Sishencong (EXHN1), at the four sides 1 Cun apart from Baihui (GV20)”. Obviously, Sishencong (EX-HN1) is extended from Baihui (GV20), and its therapeutic intent is similar as Baihui (GV20). As a result, the original structural ambit of Baihui (GV20) is far downsized due to the creation of Sishencong (EX-HN1).

Acupoint has a multidimensional structure, and the acupoint locations make a difference in their structural ambits, yet the sizes of acupoints are vague due to the fact that there are no clear boundaries for their ambits. From the historical development point of view, the bone measurement and location of each acupoint have evolved into something concrete from ambiguity, turned into precision from roughness. Unfortunately, there is no further discussion on the deeper-layer structure of acupoints by the ancients, and thus all the size range of acupuncture points is merely a relative concept. We should not limit our recognition of an acupoint as a pure shape of round dots, there is not yet any concrete quantification of the size ranges for acupoint.

\section{See the Ambiguous Nature of Acupoint's Size from Acupuncture Manipulation}

In the Spiritual Pivot: Application of Needles, there are many types of puncturing and manipulation methods, revealing the ambiguity in regard to the size ranges of acupoints.

Jingci (meridian needling): "Jingci means to puncture the large channel in conjunction with the collateral." Luoci (collateral needling): "Luoci means to puncture the minute collaterals to let out blood.” Fenci (separate needling): "Fenci means to puncture the gap between muscles.” Maoci (leather needling): "Maoci means to puncture the skin, which contains the Fubi (floating Bi-syndrome)." Huici (extensive needling): "Huici means to puncture surround the spasm muscle, lift back and forth to relax the Jinbi (Bi-syndrome of tendon).” Zhizhenci (direct needling): "Zhizhenci means to nip up the muscle and puncture directly into it, to treat the cold disease hidden at a shallow level of a body." Shuci (transmitted needling): "Shuci means to puncture perpendicularly with fewer acupoints, and insert deeply to treat the overabundant Qi and the severed heat.” Duanci (gradual needling): "Duanci is used to treat Gubi (Bi-syndrome of bone), puncture deeply reaching the bone, shake slightly up and down the bone level.” Fuci (floating needling): "Fuci means to puncture superficially surround the point to treat the cold and spasm muscles.” These puncturing methods are decided upon the locations and structures of acupoints when treating different diseases.

Pangzhenci (adjacent needling): "Pangzhenci means to insert one needle perpendicularly into the affected part along with another one on the side, to fix the obstinate Bi-syndrome.” Qici (multiple needling): “Qici means to insert one needle perpendicular into the affected part along with two others on the sides, to treat trivial cold with deeper penetration.” Yangci (scattered needling): "Yangci means to insert one needle shallowly into the center of the affected part surrounded with four others on the sides to relieve excessive Cold-Qi (syndrome)." These are the practice based on the size ranges of acupoints when deciding on the number of needles to be used: two, three or five pieces on puncturing.

Sanci (triple needling): "The so called 'Sanci is to induce Gu-Qi (Food-Qi)’ by first puncturing into the skin layer to dissipate Yang-Xie (Yang pathogen); 'Then further on to induce Yin-Xie (Yin pathogen)' by going deeper into the muscle before reaching muscular interstice; further into the muscular interstice, then Gu-Qi comes. Therefore, the book Needling Methods had said, 'First puncture shallowly to expel Xie-Qi (pathogens) with promoting blood circulation, then puncture deeper to discharge Yin-Xie, finally puncture the deepest to conduct Gu-Qi.' This is reason for what has been men- 
tioned." It has indicated that the depth of puncturing is divided into three layers, namely shallow, medium and deep puncturing, in accordance to the depth of an acupoint within the skin, underneath the skin and in the muscular interstice.

Wuci: "There are five puncturing methods that correspond to Five Zang-organs. The first one is called Banci (half-needling), which punctures superficially without making any damage to the muscles, and withdraws rapidly as if pulling out hairs, to deploy the Qi from the skin, and this is the way of needling that corresponds to the lungs. The second one is called Baowenci (leopard spot needling), which punctures to the left, right, anterior and posterior of the channel level of the treated part for bloodletting, and this is the way of needling that corresponds to the heart. The third one is called Guanci (joint needling), which punctures directly to the joints on four limbs as well as the parts on distal tendons to treat Jinbi (Bi-Syndrome of tendon), and this is the way of needling that corresponds to the liver. The forth one is called Heguci (tri-directional needling), which works like a chicken talon with needles puncturing into the muscular interstices to heal Jibi (Bi-syndrome of muscle), and this is the way of needling that corresponds to the spleen. The fifth one is called Shuci (transmitted needling), which inserts and withdraws needles perpendicularly into the bone level to cure Gubi (Bi-syndrome of bone), and this is the way of needling that corresponds to the kidneys." These are the puncturing methods based on the depth of skin, muscle, channel, tendon and bone, the five layers, in response to illnesses of the lungs, heart, liver, spleen and kidneys, the five organs.

As the size ranges of acupoints and the depth of puncturing cannot be lumped under one heading, the puncturing methods also become varied.

\section{Conclusion}

The formation and development of acupoints have surely been influenced by the ancients' ambiguous way of thinking. The size and scope of an acupoint therefore has also been carrying such sense of ambiguity. The structure and size range of an acupoint are determined not only by the outlook of depression and blood vessel areas, but also its relationship with other acupoints in its vicinity. The different manipulations of puncturing recorded in the Yellow Emperor's Canon of Medicine also show the ambiguous nature of the structure and size range of an acupoint. In theory, an acupoint has been characterized as various forms, which should not be limited to a mere round dot shape. Without such understanding, an acupuncture practitioner will be limited to a large extent in clinical application and the studies on acupoints will also go into a wrong direction.

\section{REFERENCES}

[1] The Yellow Emperor's Canon of Medicine, "Plain Conversation (Copied Print),” People’s Health Publishing House, Beijing, 1956.

[2] G. F. Dou, "Four Books of Avupuncture and Moxibuation,” People’s Health Publishing House, Beijing, 1956.

[3] F. Xu, "A Complete works in Avupuncture and Moxibuation,” People's Health Publishing House, Beijing, 1958.

[4] F. M. Huang, "The A-B Canon of Acupuncture and Moxibution (Copied Print)," People's Health Publishing House, Beijing, 1956.

[5] “The Spiritual Pivot (Copied Print),” People’s Health Publishing House, Beijing, 1956.

[6] R. H. Liao, "An Integration of Avupuncture and Moxibuation,” Cathay Bookshop, Beijing, 1986.

[7] H. Y. Wang, Y. Wang, Q. Deng, et al., "Peaceful Holy Benevolent Prescriptions,” People's Health Publishing House, Beijing, 1958. 